\title{
Microbiota Thrombus Colonization May Influence Athero-Thrombosls in Hyperglycemic Patients With ST Segment Elevation Myocardial Infarction (STEMI). MARIANELLA Study
}

Celestino Sardu ( $\nabla$ drsarducele@gmail.com )

University of Campania https://orcid.org/0000-0001-5099-3790

\section{Maria Consiglia Trotta}

University of Campania Luigi Vanvitelli: Universita degli Studi della Campania Luigi Vanvitelli

\section{Biagio Santella}

University of Campania Luigi Vanvitelli: Universita degli Studi della Campania Luigi Vanvitelli Nunzia D' Onofrio

University of Campania Luigi Vanvitelli: Universita degli Studi della Campania Luigi Vanvitelli

Michelangela Barbieri

University of Campania Luigi Vanvitelli: Universita degli Studi della Campania Luigi Vanvitelli

Maria Rosaria Rizzo

Università degli Studi della Campania Luigi Vanvitelli: Universita degli Studi della Campania Luigi

Vanvitelli

\section{Ferdinando Carlo Sasso}

University of Campania Luigi Vanvitelli: Universita degli Studi della Campania Luigi Vanvitelli

\section{Lucia Scisciola}

University of Campania Vanvitelli

\section{Fabrizio Turriziani}

University of Campania Luigi Vanvitelli: Universita degli Studi della Campania Luigi Vanvitelli

\section{Michele Torella}

University Vanvitelli

\section{Michele Portoghese}

Sassari University Hospital: Azienda Ospedaliero Universitaria di Sassari

Francesco Loreni

University Vanvitelli

\section{Simone Mureddu}

Sassari University Hospital: Azienda Ospedaliero Universitaria di Sassari

\section{Maria Antonietta Lepore}

University Vanvitelli

\section{Massimiliano Galdiero}


University Vanvitelli

\section{Gianluigi Franci}

University Vanvitelli

Veronica Folliero

University Vanvitelli

Arianna Petrillo

University Vanvitelli

Lara Boatti

University Vanvitelli

Fabio Minicucci

Cardarelli Hospital: Ospedale Cardarelli

Ciro Mauro

Cardarelli

Paolo Calabrò

University Vanvitelli

Marisa De Feo

University of Campania Luigi Vanvitelli: Universita degli Studi della Campania Luigi Vanvitelli

Maria Luisa Balestrieri

University Vanvitelli

Danilo Ercolini

Federico II

Michele D' Amico

Vanvitelli

Giuseppe Paolisso

University Vanvitelli

Marilena Galdiero

Vanvitelli

Raffaele Marfellla

University Vanvitelli

Original investigation

Keywords: hyperglycemia, STEMI, primary percutaneous coronary intervention, thrombus

Posted Date: November 18th, 2020

DOI: https://doi.org/10.21203/rs.3.rs-107251/v1

License: (c) (1) This work is licensed under a Creative Commons Attribution 4.0 International License. Read Full License 


\section{Abstract}

Objectives. We examined the association of the coronary thrombus microbiota and relative metabolites with major adverse cardiovascular events (MACE) in hyperglycemic patients with ST segment elevation myocardial infarction (STEMI).

Background. Hyperglycemia during STEMI may affect both development and progression of coronary thrombus via gut and thrombus microbiota modifications.

Methods. We undertook an observational cohort study of 146 first STEMI patients treated with primary percutaneous coronary intervention (PPCl) and thrombus-aspiration (TA). Patients were clustered, based on admission blood glucose levels, in hyperglycemic ( $\geq 140 \mathrm{mg} / \mathrm{dl})$ and normoglycemic $(<140 \mathrm{mg} / \mathrm{dl})$. We analyzed gut and thrombus microbiota in all patients. Moreover, we assessed TMAO, CD40L and von Willebrand Factor (VWF) in coronary thrombi. Cox regressions were used for the association between Prevotellaspp and TMAO terziles and MACE. MACE endpoint at 1 year included death, re-infarction, unstable angina.

Results. In fecal and thrombus samples, we observed a significantly different prevalence of both Prevotellaspp and Alistipesspp. between patients with hyperglycemia $(n=56)$ and those with normal glucose levels $(n=90)$. The abundance of Prevotella increased in hyperglycemic vs normoglycemic patients whereas the contrary was observed for Alistipes. Interestingly, in coronary thrombus, the content of Prevotella was associated with admission blood glucose levels $(\mathrm{p}<0.01)$, thrombus dimensions $(p<0.01)$, TMAO, CDL40 $(p<0.01)$ and vWF $(p<0.01)$ coronary thrombus contents. Multivariate Coxanalysis disclosed a reduced survival in patients with high levels of Prevotella and TMAO in coronary thrombus as compared to patients with low levels of Prevotella and TMAO, after 1-year follow up.

Conclusions. Hyperglycemia during STEMI may increase coronary thrombus burden via gut and thrombus microbiota dysbiosis characterized by an increase of Prevotella and TMAO content in thrombi.

\section{Background}

Glycemic and coagulative disorders may play a role in both the development and progression of coronary thrombus in patients with acute myocardial infarction (AMI). However, the mechanisms are not completely understood (1). The potential role of admission hyperglycemia in modulating the gut microbiota effects in coronary thrombus development remains unclear, and only small studies with diverse methods and findings have been reported so far (2-4). Common microbiota changes observed in obesity, diabetes mellitus and dyslipidemia include a decrease in the ratio of bacteria, such as Grampositive Firmicutes to Gram-negative Bacteroidetes, as well as either an overabundance or depletion of certain species, including Prevotella (5-8). The consequent effects of these shifts include alterations in the metabolic composition of the gut and increased levels of trimethylamine $\mathrm{N}$-oxide (TMAO). TMAO, an amine oxide, issynthesized from choline, betaine and carnitine, which are transformed to trimethylamine (TMA) by human gut microbiota $(8,9)$.TMA is then oxidized to TMAO by the hepatic flavin 
monooxygenase 3 (FMO3) (10). Among the genera of gut microbiota, Prevotella has showed the strongest association with TMAO production (11). Baseline measures of 53 participants in a controlled feeding study showed that those with a Prevotella enterotype had higher plasma TMAO concentrations than those with a Bacteroides enterotype (12). Moreover, recent data showed the involvement of both Prevotella (14) and TMAO (13) in the arterial thrombosis. To date, evidence about the possibleinteraction between hyperglycemia, microbiota and its metabolitesin the development and progression of coronary thrombus in ST-elevation myocardial infarction (STEMI) patients is lacking. To define a more robust evidence of gut microbiota signature and TMAO in coronary thrombus development and progression during AMl, we assessed both gut and thrombus microbiota in hyperglycemic (blood glucose levels $>140$ $\mathrm{mg} / \mathrm{dl}$ at admission) and normoglycemic patients with STEMI. Furthermore, we explored associations between thrombus microbiota and TMAO levels, and composite endpoints (MACE= death, re-infarction, unstable angina) in cohorts at 1-year.

\section{Materials And Methods}

\section{Patients and study design}

This was a multicenter observational prospective cohort study aimed to investigate the relationship between gut microbiota signature, TMAO thrombus levels, and 1-year outcomes in STEMI patients. We examined an observational cohort of consecutive patients with first STEMI treated with PPCl and thrombus aspiration (TA), between February 2016 and September 2019 at the Department of Cardiology of Cardarelli Hospital in Naples Italy, at Department of Cardiology of the University of Campania "Luigi Vanvitelli" Italy, and at Department of Cardiac Surgery of the SS. Annunziata Hospital, Sassari, Italy. All patients with onset of symptoms $<12 \mathrm{~h}$ and at least 1-mm ST-segment elevation in 2 or more contiguous limb leads or at least $2 \mathrm{~mm}$ in 2 or more contiguous precordial leads or left bundle branch block were considered eligible for PPCI. Coronary angiography was performed either via the radial or femoral artery. The culprit lesion was identified and crossed with an angioplasty guidewire. Manual TA was performed at the discretion of the operator, due to technical aspects (e.g., type and number of stents, use of any other devices), and with consideration of angiographic selection criteria (e.g., the presence of a visible thrombus on angiography), followed by conventional PCI to the culprit vessel. We considered eligible for the study all patients with: correspondence between ECG findings and suspected culprit artery; a minimum visual estimate of $50 \%$ stenosis in the culprit artery, and feasibility of performing TA, as judged by the treating physician; age of 18 years or greater; presentation to the cardiac catheterization laboratory for PPCl in the setting of first STEMI. Patients with left ventricular ejection fraction less than $25 \%$, with previous myocardial infarction or previous $\mathrm{PPCl}$ and/or coronary by-pass grafting, or who had received fibrinolytic therapy were instead excluded from the study.

Based on the admission blood glucose levels, patients were clustered in two groups: (a)hyperglycemic STEMI patients (glucose levels $\geq 140 \mathrm{mg} / \mathrm{dl}$ ), as suggested by the position statement of the American Heart Association (ADA) (15) and normoglycemic STEMI patients (glucose levels $<140 \mathrm{mg} / \mathrm{dl}$ ). The study is in accordance with the principles outlined in the 1976 the Declaration of Helsinki and its later 
amendments for use of human tissue or subjects. The Institutional Review Board of University of Campania “Luigi Vanvitelli” Italy approved the protocol.

\section{Procedures}

Routine analyses were obtained on admission before coronary angiography and before the initiation of full medical therapy. Stool samples were instead obtained during the first day after admission. Treatment was classified as "thrombus aspiration" when TA was attempted as suggested by the operator, irrespective of procedure success. Thrombus grade was classified in accordance with Sianos et al. (16) into: Grade 0 (G0), no angiographic characteristics of thrombus present; Grade 1 (G1), possible thrombus present, with the following angiography characteristics: reduced contrast density, haziness, irregular lesion contour, or a smooth convex meniscus at the site of total occlusion suggestive, but not diagnostic, of thrombus; Grade 2 (G2), definite thrombus with largest dimension $\leq 1 / 2$ the vessel diameter; Grade 3 (G3), definite thrombus, with largest linear dimension > $1 \frac{1}{2}$ but <twice vessel diameter; Grade 4 (G4), definite thrombus, with the largest dimension $\geq 2$ vessel diameters; Grade 5 (G5), total occlusion, unable to assess thrombus burden due to total vessel occlusion. Detailed recommendations about TA procedure were further provided. The procedure was done by interventional cardiologists' investigators. All patients were treated with adenosine (given $120 \mathrm{mcg}$ as a fast bolus followed by $2 \mathrm{mg}$ in 2 minutes) and with bolus infusion of Abciximab $(0.25 \mathrm{mg} / \mathrm{kg}$ IV bolus). Patients undergoing TA received the same treatment also after thrombus aspiration. Aspiration had to be started before crossing the lesion, with a minimum of two syringes $(40 \mathrm{~mL})$ of aspirate recommended. Investigators were appropriately trained to ensure that the guide catheter was engaged with the coronary ostia when removing the thrombectomy catheter. Finally, the guide catheter was aspirated after thrombectomy to avoid embolization of either air or thrombus from the guide catheter.

\section{Stool sample collection and fecal microbiome analysis}

Stool sample was collected in a clean container and immediately stored at $-20^{\circ} \mathrm{C}$ and processed within 48 hours. The $-20^{\circ} \mathrm{C}$ stool samples were defrosted. $220 \mathrm{mg}$ of dried stool were suspended in 1,4 ml transport buffer by vortexing and transferred to a sterile DNase/RNase-free $2.0 \mathrm{~mL}$ tube for enzymatic lysis. Total DNA was extracted from stool sample using a DNA isolation kit (QIAamp® DNA Stool Handbook kit, cat. no. 51504; Qiagen $®$, Germany), according to the manufacturer's protocols. The total genomic DNA quality was assessed by $1,8 \%$ agarose gel electrophoresis, and the DNA concentration measured using to the Qubit fluorometer with the dsDNA HS (high sensitivity) Assay Kit (Invitro-gen Q32854) to evaluate DNA yield (ng) and normalized to $1 \mathrm{ng} / \mu \mathrm{L}$.

Illumina MiSeq sequencing of 16S rRNA gene amplicons. The V3-V4-V6 hypervariable regions of the 16S rRNA gene were amplified using the Arrow Microbiota Solution B kit (cod. AD-002.024). Amplification was performed in 20- $\mu$ l reactions with amp mix for "PCR target" and $5 \mu \mathrm{L}$ of DNA extract. The reactions were performed using a SimpliAmp Thermal Cycler (Applied Biosystems ${ }^{\circledR}, \# A 24812$ ) under the following thermal profile: $95^{\circ} \mathrm{C}$ for $5 \mathrm{~min}$, followed by 30 cycles of $95^{\circ} \mathrm{C}$ for $30 \mathrm{~s}, 55^{\circ} \mathrm{C}$ for $30 \mathrm{~s}$, and $72{ }^{\circ} \mathrm{C}$ for $30 \mathrm{~s}$, and one cycle of $72{ }^{\circ} \mathrm{C}$ for 5 min and a $4{ }^{\circ} \mathrm{C}$ hold. PCR products were examined by $1,6 \%$ agarose gel 
electrophoresis and then purified using AMPure Beads XP (cat. n. A63880, Beckman Coulter, Inc.). The amplicons quality was assessed by $1,6 \%$ agarose gel electrophoresis, product visible as a band with a molecular weight of approximately $840 \mathrm{bp}$. A 6-bp barcode sequence was added to the ends of both forward and reverse primers in the PCR "index". PCR "index" products were examined and then purified as described above. Amplicons targets were normalized to $10 \mathrm{nM}$ and used to prepare the library for sequencing. The library was processed using kit MiSeq Reagent Nano Kit v2 (500-cycles) (cat. n: MS-1031003, Illumina Inc.), following the user guidelines for the system MiSeq Illumina ("Denature ana Dilute Libraries Guide"-MiSeq System). The library was corrected by adding 5\% of Phix Control v3 (cat. n. FC110-3001, Illumina Inc.) and sequenced using an Illumina MiSeq platform. The amplicons were pooled, and sequencing was conducted at Ames Center (Naples, Italy), with an Illumina MiSeq sequencing system (Illumina, San Diego, CA, USA). Obtained data were processed with the MicrobAT (Microbiota Analysis Tool) system of the SmartSeq S.r.l.

$16 S$ rRNA gene sequence analysis. Sequence analysis of low quality with a lower quality score of 20 , a length less than $50 \mathrm{bp}$, or any misalignments to the primer or barcode containing chimeras were removed.

\section{Thrombus analysis}

Thrombi obtained from matched STEMI patients were analyzed for microbiota colonization, TMAO levels and pro-thrombotic molecules. All analyses were performed by 3 independent pathologists, blinded to the patients' characteristics. After TA, the specimens were frozen in liquid nitrogen, stored at $-20^{\circ} \mathrm{C}$ and processed within 48 hours. After defrosted, the specimens were cut perpendicular to the long axis into two halves. The first half was used for the following ELISA analysis: CD40L (Soluble- Human ELISA Kit, Invitrogen); von Willebrand Factor(VWF) (Human von Willebrand Factor ELISA Kit - ab223864); TMAO (TMAO elisa kit: Human Trimethylamine-N-oxide Reductase ELISA Kit MBS7254766, MyBiosource). A portion of the other half of the specimen was instead analyzed for microbiota colonization as previously described for stool samples.

\section{In vitro studyof bacterial growth.}

In order to analyze the growth response of Prevotella and Bacteroides toward different concentrations of glucose, bacterial strains were grown as previously described (17). Briefly, Prevotella and Bacteroides from their glycerol stocks were firstly cultured on blood agar plates, then single colonies were picked individually and cultured in an anaerobic chamber at $37^{\circ} \mathrm{C}$ overnight in Gifu Anaerobic Medium $\left(\mathrm{OD}_{600} 1.0-1.5\right)$. Bacterial cultures were centrifuged at $1300 \times \mathrm{g}$ for $5 \mathrm{~min}$ and culture pellets were washed. Twenty microliters of the culture were then added to $2 \mathrm{ml}$ medium supplemented with different concentrations of D-glucose, $7 \mathrm{mM}, 14 \mathrm{mM}, 22 \mathrm{mM}$ and $32 \mathrm{mM}$. Measures of optical density at $600 \mathrm{~nm}$ wavelength at $0,12 \mathrm{~h}, 24 \mathrm{~h}, 36 \mathrm{~h}, 48 \mathrm{~h}, 60 \mathrm{~h}$ and $72 \mathrm{~h}$ were performed in order to assess bacterial growth rates. All experiments were performed in triplicate. One-way analysis of variance with Tukey's test was performed using GraphPad Prism (version 6 for Windows, GraphPad Software, La Jolla, CA, USA). 


\section{Blood glucose control in emergency wards.}

After coronary angiography procedures, all hyperglycemic patients with blood glucose $>180 \mathrm{mg} / \mathrm{dl}$ were treated with intensive glucose control to keep blood glucose levels between 140 and $180 \mathrm{mg} / \mathrm{dl}$, as previously described (18). Continuous insulin infusion of $50 \mathrm{IU}$ Actrapid HM (Novo-Nordisk) in $50 \mathrm{ml} \mathrm{NaCl}$ (0.9\% using a Perfusor-FM-pump) was started only when blood glucose levels exceeded $180 \mathrm{mg} / \mathrm{dl}$, and adjusted to keep blood glucose between 140 and $180 \mathrm{mg} / \mathrm{dl}$. When blood glucose fell $<140 \mathrm{mg} / \mathrm{dl}$, insulin infusion was tapered and eventually stopped. After the start of insulin infusion protocol, a glycemic control was provided every hour in order to obtain three consecutive values that were within the goal range. The infusion lasted until achievement of stable glycemic goal (140-180 mg/dl) for at least $24 \mathrm{~h}$. Once the glycemic goal was preserved for $24 \mathrm{~h}$, the infusion was stopped, and subcutaneous insulin was initiated. Insulin was given as short-acting insulin before meals and long-acting insulin in the evening throughout the whole hospitalization period. As for the full medical therapy, the protocol stated that the use of concomitant treatment should be as uniform as possible and in accordance with evidence-based international guidelines for STEMI (19).

\section{Follow-up}

After discharge, all patients were managed and followed quarterly for 12 months after event, as outpatients, to perform clinical evaluation, routine analyses and cardiovascular evaluation (ECG, exercise ECG, echocardiography), alongside with the objective to keep HbA1c levels $<7 \%$ in diabetic patients.

\section{Outcomes}

The outcome: clinical outcomes at 1-year follow-up were major adverse cardiovascular events (MACEs). MACEs are defined as a composite of cardiovascular death, non-fatal acute coronary syndrome and heart failure.

\section{Statistical analyses}

After appropriate assessment of their normal distribution by the Shapiro-Wilk test, differences between continuous variables were analyzed either with the parametric Student t-test or the non-parametric MannWhithney $U$ test. Differences between categorical variables were instead evaluated by the Chi-square $(\chi 2)$ test. $P$ values $<0.05$ were considered statistically significant. The study population was equally distributed for age $(p=0.807)$. To evaluate the independent contribution of hyperglycaemia in thrombus Prevotella and TMAO levels, we performed a correlation between Prevotella and TMAO levels in coronary thrombus and blood glucose level during STEMI. Risk adjusted Cox-regression analysis curves showing survival from severe disease through days of hospitalization. Cox models were adjusted for; age, diabetes, hypertension, diabetes, new diabetes, dyslipidemia, cigarette smoking, BMI, systolic blood pressure, total cholesterol, HDL and LDL-cholesterol levels, triglycerides, TIMI scores, lesions of RCA and LM, dual antiplatelet therapy and ejection fraction. To investigate the effects of Prevotella and TMAO thrombi expressions on cardiovascular endpoints, we evaluated STEMI outcomes at 1-year follow-up 
stratified by Prevotella and TMAO terziles. Statistical analysis was performed by SPSS 23 computer program.

\section{Results}

Patient characteristics. 678 patients with first STEMI (106 with hyperglycemia) were admitted at cardiologic study centers between January 2016 to July 2019. Of these, 38 patients were excluded from the study, 26 due to an onset of symptoms longer than $12 \mathrm{~h}$ and 12 as they died before angiographic study. Consequently, 640 were considered eligible STEMI patients and underwent to the angiographic study (91 with hyperglycemia). After coronary study, 101 patients were excluded due to CABG indication, 11 for mental disorders, 21 for absence of coronary lesions, 99 for incomplete data, whilst 355 were not treated with TA. Thus, 152 patients treated with PPCI and TA were definitely enrolled in the study, of which 59 with hyperglycemia (38.8\%) (Supplementary figure 1). During the follow-up, 3 normoglycemic patients and 3 hyperglycemic patients voluntarily withdraw from the study and/or were excluded due to incomplete data. Therefore, the final study cohort included 90 normoglycemic and 56 hyperglycemic patients followed for 1 year. Hyperglycemic patients had higher plasma cholesterol, low-density lipoprotein (LDL), lower high-density lipoprotein (HDL) levels and thrombus grade (Table 1). No differences in the troponin levels, in the median time between symptom onset and the start of angiography procedure at hemodynamic unit, in the number of coronary vessel diseases and in the number of patients with post-procedural TIMI 3 were observed (Table 1).

In-hospital treatments and glucose control. During hospitalization, hyperglycemic patients were treated according to the most recent international guidelines (23), with high proportions of patients receiving platelet inhibitors and antithrombotic agents before, during and after the procedure. In particular, aspirin was administered to $98 \%$ of patients and statins to $86 \%$ in both groups. Beta-blockers were given to $81 \%$ of patients, of whom the $43 \%$ received an ACE-inhibitor in both groups. Among the whole study population, $88 \%$ of patients were treated with a combination therapy of thienopyridine and aspirin. After $\mathrm{PPCl}$, the glycemic goal was preserved for $24 \mathrm{~h}$ (mean glycemia $160 \pm 23 \mathrm{mg} / \mathrm{dl}$ ). In the $10 \%$ of patients, blood glucose $<70 \mathrm{mg} / \mathrm{dl}$ both with and without symptoms occurred during insulin infusion. At hospital discharge, both fasting and post-prandial plasma glucose levels were: fasting, $133 \pm 31 \mathrm{mg} / \mathrm{dl}$, postprandial $177 \pm 19 \mathrm{mg} / \mathrm{dl}$. In addition, the rate of ACE-inhibitors, aspirin, beta-blockers, statins, and thienopyridines use increased as compared to that on admission both in normoglycemic and hyperglycemic patients (Table 1).

\section{Fecal microbiota}

Differences between gut microbiota of hyperglycemic and normoglycemic STEMI patients were observed (Figure 1). Some bacterial taxa in the intestinal microbiota were found significantly more abundant in patients with hyperglycaemia as compared to normoglycemic patients. At the Phylum level, the Bacteroitedes are more abundant in hyperglycemic compared to normoglycemic patients ( $46.5 \% \mathrm{vs}$. $21.5 \% ; P=0.007$ ) (Supplementary figure 2). However, the Firmicutes and the Actinobacteria were reduced 
in hyperglycemic compared to normoglycemic patients (Firmicutes: $37.1 \%$ vs $62.1 \%: P=0.003$ ) (Actinobacteria: $3.4 \%$ vs $10 \%, P=0.001$ ). At the genus level, the enriched proportions belong to the genus Prevotella increased by 3-fold while the Bacteroides were reduced by 10-fold in hyperglycemic compared to normoglycemic patients (Supplementary figure 2). Examination of the percentage of specific genera and bacterial species present in stool revealed that only few genera differed between hyperglycemic and normoglycemic patients (Supplementary figure 2). Among Prevotella genus, Prevotella DJF RP53 was almost 5-fold higher in hyperglycemic as compared to normoglycemic patients (Supplementary figure 2).

Within the Bacteroidetes, Alistipes was less represented in fecal sample from hyperglycemic patients, conversely to the well depiction in normoglycemic patients $(0.51 \%$ vs $4.2 \%, P<0.05)$. Finally, fecal Prevotella content was significantly associated with admission blood glucose levels $(p<0.01)$

\section{(Supplementary figure 2).}

\section{Thrombus microbiota}

Differences between thrombus microbiota of hyperglycemic and normoglycemic STEMI patients are reported $(p<0.001)$ (Figure 2 ). Bacteroitedes and Proteobacteria are more abundant in the hyperglycemic rather than normoglycemic patients (Bacteroitedes: 44.6\% vs 19.5\%, $\mathrm{P}=0.011$ and Proteobacteria: $22.6 \%$ vs $7.6 \%, \mathrm{P}=0.001)$. On the contrary, Firmicutes and Actinobacteria show a reduced prevalence among hyperglycemic as compared to normoglycemic patients (Firmicutes: $26.1 \%$ vs $60.9 \%$, $P=0.005$ and Actinobacteria: $9.9 \%$ vs 33.5\%, $P=0.001$ ) (Figure 2). At the genus level, the highest rates belong to the genus Prevotella, with a 5-fold increase, while Bacteroides disclosed a 7-fold reduction in hyperglycemic as compared to normoglycemic patients (Figure 2). Examination of the prevalence of specific genera and bacterial species present in thrombus revealed that few genera can be significantly different between hyperglycemic and normoglycemic patients (Figure 2). Alistipes was absent in hyperglycemic thrombi, whilst it occurred in normoglycemic patients (7.3\%). Finally, thrombus Prevotella content was significantly associated with admission blood glucose levels ( $P=0.001)$ (Figure 2).

\section{In-vitro study, effects of high glucose concentration on growth curve of Prevotella DJF RP53 and} Alistipes. Due to the major modification of Prevotella DJF RP53 and Alistipes in hyperglycemic as compared to normoglycemic thrombi, we assessed the effects of high-glucose concentration on Prevotella DJF RP53 and Alistipes growth curve on soil. Results clearly indicated that Prevotella DJF RP53 growth curve was accelerated and increased in soil containing high-glucose concentration, as compared with normal glucose concentration $(\mathrm{P}=0.012)$. On the other hand, high-glucose concentration slowed and decreased the Alistipes growth curve on soil, as compared normal glucose concentration on soil ( $P=0.016)$ (Figure 3 ).

\section{Coronary thrombus analysis in hyperglycemic and normoglycemic patients}

The volume and size of the thrombi, and the number of retrieved fragments were significantly higher in hyperglycemic as compared to normoglycemic patients $(P<0.001)$ (Supplementary figure 3 ). Thrombi composition displayed higher CD40L, VWF and TMAO levels as compared to normoglycemic patients 
(Supplementary figure 2). Moreover, microbiota genus analysis revealed a marked presence of Prevotella in hyperglycemic thrombi which,instead, was almost absent in normoglycemic thrombi (Supplementary figure 2). Moreover, Prevotella thrombus levels were significantly associated with the size of thrombi, and TMAO, CDL40 and VWF levels during STEMI (Figure 4).

\section{Treatments at 12 months following discharge}

Between presentation of STEMI and the 12 months of follow-up after hospital discharge, 16 patients $(10.9 \%)$ in the entire study cohort disclosed a new onset of diabetes, of which 15 (26.8\%)among hyperglycemic patients. Diabetic patients did not show any significant difference in metabolic control between groups in overall population (Table 1). Over the 1-year follow-up period, there was no difference in both anti-diabetic and cardiovascular therapies between the two groups. During 1-year follow up, there are no differences in glycemic control among the groups (data not shown)

Post-discharge outcomes. After 12 months following PPCl, a multivariable Cox regression analysis, adjusted for age, diabetes, hypertension, diabetes, new diabetes, dyslipidemia, cigarette smoking, BMI, systolic blood pressure, total cholesterol, HDL and LDL-cholesterol levels, triglycerides, TIMI scores, lesions of RCA and LM, dual antiplatelet therapy and ejection fraction, showed a significantly higher oneyear overall survival for MACE in normoglycemic patients (Figure 5). STEMI outcomes at 1-year follow-up stratified by Prevotella and TMAO level terziles in coronary thrombi of hyperglycemic and normoglycemic patients were further assessed to translate molecular results in clinical endpoints. Cox multivariable regression analysis reported a lower survival from events among patients with higher Prevotella and TMAO levels (Figure 5). Interestingly, we observed an increased risk association with Prevotella and TMAO terziles, consistent regardless of the dual antiplatelet therapy during the follow-up, thus suggesting a reduction of effectiveness of anti-platelet therapy in patients with high levels of Prevotella and TMAO thrombus levels.

\section{Discussion}

In the present study we investigated for the first time the relationship between thrombus Prevotella colonization and recurrent acute coronary syndrome and death among hyperglycemic patients with first STEMI. Moreover, we analyzed the relationship between thrombus microbe colonization and thrombus size, TMAO content, as well as thrombus levels of CD40L and VWF. We observed significant differences between gut and thrombus microbiota of hyperglycemic and normoglycemic STEMI patients. Fecal and thrombus Prevotella contents were more abundant in hyperglycemic than normoglycemic patients and, interestingly, were also significantly associated with admission blood glucose levels. On the contrary, Alistipes contents were lower in fecal samples and absent in thrombus samples from hyperglycemic patients, while well represented in normoglycemic patients.

These findings raise two important considerations. First, hyperglycemia on admission, in the setting of acute coronary syndrome, may suggest a substantial change in both gut and coronary thrombus microbiota compositions. In fact, fecal and thrombus samples from hyperglycemic patients showed 
higher contents of Prevotella and low Alistipes contents as compared to samples from normoglycemic patients. This hypothesis is supported by data from our in vitro study. Indeed, the high-glucose concentration, mimicking the hyperglycemic milieu, accelerated and increased Prevotella DJF RP53 growth curve and slowed and decreased Alistipes growth curve on soil. In addition, during acute coronary syndrome, hyperglycemia may favor thrombus colonization, thus driving intestinal barrier permeability (20). In this setting, hyperglycemia and GLUT2-dependent transcriptional reprogramming alter both tight and adherence junction integrity of intestinal epithelial cells (20). Consequently, hyperglycemia-mediated barrier disruption leads to the systemic influx of microbioma and an enhanced systemic dissemination. Moreover, treatment of hyperglycemia, intestinal epithelial-specific GLUT2 deletion, or inhibition of glucose metabolism restore barrier function and bacterial containment (20). Thus, our data suggest that the increase in the intestinal barrier permeability among patients with hyperglycemia may favor thrombus contamination in a coronary syndrome setting.

The role of both Prevotella and Alistipes in the metabolic and cardiovascular diseases has been already reported by previous studies (21).Fecal and gingival Prevotella increased both in diabetic and hyperglycemic patients as compared to healthy subjects $(22,23)$. These studies not only demonstrated a positive association of Prevotella gingival content with higher blood glucose levels, though also a significant contribution of Prevotella gingival content to the development of disease condition, via increased TMAO production (23). Accordingly, data from recent studies (11) suggest that Prevotella, among the microbialgenera, showed the strongest association with TMAO levels. On the contrary, abundance of stool Alistipes has been associated with low levels of TMAO (24). There is considerable evidence linking the metaorganismal TMAO pathway to the development of atherosclerosis and thrombosis in animal models and incident cardiovascular disease risks. In particular, the proatherogenic effects of microbe-dependent TMAO formation include enhanced macrophage cholesterol accumulation and subsequent foam cell formation, proinflammatory changes in the artery wall, and promotion of both platelet hyperactivity and enhanced arterial thrombosis potential. (25). Thus, our data suggest a potential antagonistic effect between Alistipes and Prevotella, as shown by the absence of Alistipes and higher contents of Prevotella in hyperglycemic coronary thrombus, in the setting of acute coronary syndrome. This trend has been commonly seen in other heart conditions, such as atrial fibrillation and heart dysfunction $(26,27)$. Moreover, to date, there has been no evidence about the possible role of Prevotella thrombus colonization in outcomes of high-risk patients (e.g., those found in hyperglycemic patients during STEMI), and about the specific pathways transducing thrombus Prevotella stimuli in the modulation of coronary thrombus burden.

The novelty of our study is represented by the evidence that Prevotella coronary thrombus content is remarkably increased and it is associated with high thrombus burden, TMAO, CDL40, and VWF in hyperglycemic patients as compared with normoglycemic patients with STEMI. Interestingly, Prevotella coronary thrombus contents were associated with admission blood glucose levels. These data suggest that high thrombus burden observed in hyperglycemic patients could represent a result of the Prevotella effects on increased TMAO levels and on the consequent effects on CDL40 and vWF thrombus contents, as reported in experimental studies that clearly underlined the role of TMAO in increasing coagulation 
(13). In addition, several studies (28) suggest that fecal over-abundance of Prevotella is associated with an increase in insulin-resistance. The role of Prevotella in the human microbiome is controversial, and the relative abundance of Prevotella in general and $P$. copri is highly dependent on diet and lifestyle (29-31). We can speculate that hyperglycemia could activate the following harmful vicious circle: I) hyperglycemia promotes systemic diffusionof Prevotella; II) Prevotella overabundance associates with increased TMAO levels; III) high TMAO levels increase systemic IR; IV) increased insulin-resistance in turn increases hyperglycemia (Figure 6). This hypothesis may have a clinical implication since patients with higher Prevotella and TMAO thrombus contents were more likely to experience subsequent cardiovascular events and death. The associations observed between Prevotella and TMAO thrombus contents and cardiovascular events and death remained significant also after adjustment for potentially confounding clinical factors. Consistently with such evidence, in a contemporary sample of patients with STEMI treated with PPCl and TA in routine practice, we observed a higher cumulative incidence of 1-year mortality and adverse cardiovascular outcomes, in patients with higher thrombus Prevotella and TMAO contents. In fact, the 1-year prognosis of patients with STEMI and III terziles of Prevotella $(<2.72 \%)$ and TMAO (>60 ng/ml) was significantly worse, with a $12.1 \%$ rate of death or re-admission for cardiovascular diseases, as compared with prognosis of patients with STEMI and I terziles of Prevotella $(>1.25 \%)$ and TMAO $(>36 \mathrm{ng} / \mathrm{ml})$, which disclosed a $1.9 \%$ rate of death or re-admission due to cardiovascular diseases at follow-up. TMAO levels are also affected by diet in a microbiome-driven manner, and lower levels were associated with consumption of plant-based diets (32) although an anticipated reduction of TMAO levels was not observed upon intervention with high-vegetables Mediterranean diet in obese subjects (33).Interestingly, the 1-year follow-up results based on Prevotella and TMAO thrombus contents did not change despite a similar severity of atherosclerotic disease at baseline and asimilar glycemic control both at discharge and at follow-up. These observations suggest that on admission hyperglycemia may play a pivotal role in the increase of coronary thrombus burden, during STEMI, favoring thrombus colonization and proliferation by Prevotella.

However, our study has some limitations. First, the observational prospective study design and the relatively small sample size. In addition, no power calculation could be performed, due to the lack of previous studies in this setting. As a consequence, the enrollment of patients in each group was arbitrary.

\section{Conclusion}

In conclusion, our data suggest that the coronary thrombus environment is more likely susceptible to a microbial colonization which might stimulate a pro-coagulatory response in acute coronary syndrome. These findings add new elements to the pathogenesis of ACS and suggest novel therapeutic targets.

\section{Abbreviations}

microbiota; TMAO.

Abbreviations: 
$H D L=$ high-density lipoprotein;

LDL= low-density lipoprotein;

MACE = major adverse cardiovascular events;

$\mathrm{PPCl}=$ primary percutaneous coronary intervention;

$\mathrm{RCA}=$ right coronary artery;

STEMI= ST-segment elevation myocardial infarction;

$\mathrm{TA}=$ thrombus aspiration;

TMAO $=$ Trimethylamine- $\mathrm{N}$-oxide.

vWF $=$ von Willebrand Factor

\section{Declarations}

Ethics approval and consent to participate: Ethical Committees of all participating Institutions gave full consent to participate in the study. Ethical Committee number 268/2016.

Consent for publication: all authors gave full consent for study publication.

Availability of data and materials: data and materials are fully available.

Competing interests:

Funding: This research received a specific grant from PRIN 2017.

Authors' contributions: C.S, M.C.T, R.M, G.P and M.G: study writing, editing and data interpretation; B.S, N.DO, M.B, M.R.R, F.C.S, M.DA, M.G: data analysis and interpretation; L.S, F.T: data collection; M.T, M.P, F.L, S.M, M.A.L, A.B, A.R, D.DA, F.M, C.M, P.C, M.DF, D.E: performed interventions and samples collection.

Acknowledgements: all authors have equally contributed to this research.

Conflict of interest: none.

Clinical Trial Registration: NCT03439592. September 30, 2016

\section{References}

1. Pretorius L, Thomson GJA, Adams RCM, Nell TA, Laubscher WA, Pretorius E. Platelet activity and hypercoagulation in type 2 diabetes. Cardiovasc Diabetol. 2018 Nov 2;17(1):141. 
2. D'Onofrio N, Sardu C, Paolisso P, Minicucci F, Gragnano F, Ferraraccio F, Panarese I, Scisciola L, Mauro C, Rizzo MR, Mansueto G, Varavallo F, Brunitto G, Caserta R, Tirino V, Papaccio G, Barbieri M, Paolisso G, Balestrieri ML, Marfella R. MicroRNA-33 and SIRT1 influence the coronary thrombus burden in hyperglycemic STEMI patients. J Cell Physiol. 2020 Feb;235(2):1438-1452.

3. Sardu C, D'Onofrio N, Mauro C, Balestrieri ML, Marfella R. Thrombus aspiration in hyperglycemic patients with high inflammation levels in coronary thrombus. J Am Coll Cardiol. 2019 Feb 5;73(4):530-531.

4. Sardu C, Barbieri M, Balestrieri ML, Siniscalchi M, Paolisso P, Calabrò P, Minicucci F, Signoriello G, Portoghese M, Mone P, D'Andrea D, Gragnano F, Bellis A, Mauro C, Paolisso G, Rizzo MR, Marfella R. Thrombus aspiration in hyperglycemic ST-elevation myocardial infarction (STEMI) patients: clinical outcomes at 1-year follow-up. Cardiovasc Diabetol. 2018 Nov 29;17(1):152.

5. Sohail MU, Althani A, Anwar H, Rizzi R, Marei HE. Role of the Gastrointestinal Tract Microbiome in the Pathophysiology of Diabetes Mellitus. J Diabetes Res. 2017;2017:9631435.

6. Brunkwall L, Orho-Melander M. The gut microbiome as a target for prevention and treatment of hyperglycaemia in type 2 diabetes: from current human evidence to future possibilities. Diabetologia. 2017 Jun;60(6):943-951.

7. Tang WH, Kitai T, Hazen SL. Gut Microbiota in Cardiovascular Health and Disease. Circ Res. 2017 Mar 31;120(7):1183-1196.

8. Heianza Y, Sun D, Smith SR, Bray GA, Sacks FM, Qi L. Changes in Gut Microbiota-Related Metabolites and Long-term Successful Weight Loss in Response to Weight-Loss Diets: The POUNDS Lost Trial. Diabetes Care. 2018 Mar;41(3):413-419.

9. Li XS, Obeid S, Klingenberg R, Gencer B, Mach F, Räber L, Windecker S, Rodondi N, Nanchen D, Muller O, Miranda MX, Matter CM, Wu Y, Li L, Wang Z, Alamri HS, Gogonea V, Chung YM, Tang WH, Hazen SL, Lüscher TF. Gut microbiota-dependent trimethylamine N-oxide in acute coronary syndromes: a prognostic marker for incident cardiovascular events beyond traditional risk factors. Eur Heart J. 2017 Mar 14;38(11):814-824.

10. Wang Z, Klipfell E, Bennett BJ, Koeth R, Levison BS, Dugar B, Feldstein AE, Britt EB, Fu X, Chung YM, Wu Y, Schauer P, Smith JD, Allayee H, Tang WH, DiDonato JA, Lusis AJ, Hazen SL. Gut flora metabolism of phosphatidylcholine promotes cardiovascular disease. Nature. 2011 Apr 7;472(7341):57-63.

11. Fu BC, Hullar MAJ, Randolph TW, Franke AA, Monroe KR, Cheng I, Wilkens LR, Shepherd JA, Madeleine MM, Le Marchand L, Lim U, Lampe JW. Associations of plasma trimethylamine N-oxide, choline, carnitine, and betaine with inflammatory and cardiometabolic risk biomarkers and the fecal microbiome in the Multiethnic Cohort Adiposity Phenotype Study. Am J Clin Nutr. 2020 Jun 1;111(6):1226-1234.

12. Chen M-I, Yi L, Zhang Y, Zhou X, Ran L, Yang J, Zhu J-d, Zhang Q-y, Mi M-t. Resveratrol attenuates trimethylamine-N-oxide (TMAO)-induced atherosclerosis by regulating TMAO synthesis and bile acid metabolism via remodeling of the gut microbiota. mBio 2016;7(2):e02210-15. 
13. Cheng X, Qiu X, Liu Y, Yuan C, Yang X. Trimethylamine N-oxide promotes tissue factor expression and activity in vascular endothelial cells: A new link between trimethylamine $\mathrm{N}$-oxide and atherosclerotic thrombosis. Thromb Res. 2019 May;177:110-116.

14. Kasselman LJ, Vernice NA, DeLeon J, Reiss AB. The gut microbiome and elevated cardiovascular risk in obesity and autoimmunity. Atherosclerosis. 2018 Apr;271:203-213.

15. Deedwania P, Kosiborod M, Barrett E. American Heart Association Diabetes Committee of the Council on Nutrition, Physical Activity, and Metabolism. Hyperglycemia and acute coronary syndrome: a scientific statement from the American Heart Association Diabetes Committee of the Council on Nutrition, Physical Activity, and Metabolism. Circulation. 2008;117:1610-1619

16. Sianos G, Papafaklis MI, Serruys PW. Angiographic thrombus burden classification in patients with ST-segment elevation myocardial infarction treated with percutaneous coronary intervention. J Invasive Cardiol 2010;22:6B-14B.

17. Pareek S, Kurakawa T, Das B, et al. Comparison of Japanese and Indian intestinal microbiota shows diet-dependent interaction between bacteria and fungi. NPJ Biofilms Microbiomes. 2019;5:37.

18. Marfella R, Rizzo MR, Siniscalchi M, Paolisso P, Barbieri M, Sardu C, Savinelli A, Angelico N, Del Gaudio S, Esposito N, Rambaldi PF, D'Onofrio N, Mansi L, Mauro C, Paolisso G, Balestrieri ML. Periprocedural tight glycemic control during early percutaneous coronary intervention up-regulates endothelial progenitor cell level and differentiation during acute ST-elevation myocardial infarction: effects on myocardial salvage. Int J Cardiol. 2013 Oct 9;168(4):3954-62.

19. 2009 Focused Updates: ACC/AHA Guidelines for the Management of Patients With ST-Elevation Myocardial Infarction (Updating the 2004 Guideline and 2007 Focused Update) and ACC/AHA/SCAI Guidelines on Percutaneous Coronary Intervention (Updating the 2005 Guideline and 2007 Focused Update). J Am CollCardiol. 2009;54:2205-41.

20. Thaiss CA, Levy M, Grosheva I, Zheng D, Soffer E, Blacher E, Braverman S, Tengeler AC, Barak O, Elazar M, Ben-Zeev R, Lehavi-Regev D, Katz MN, Pevsner-Fischer M, Gertler A, Halpern Z, Harmelin A, Aamar S, Serradas P, Grosfeld A, Shapiro H, Geiger B, Elinav E. Hyperglycemia drives intestinal barrier dysfunction and risk for enteric infection. Science. 2018 Mar 23;359(6382):1376-1383.

21. Parker BJ, Wearsch PA, Veloo ACM, Rodriguez-Palacios A. The Genus Alistipes: Gut Bacteria With Emerging Implications to Inflammation, Cancer, and Mental Health. Front Immunol. 2020 Jun 9;11:906.

22. Ejtahed HS, Hoseini-Tavassol Z, Khatami S, Zangeneh M, Behrouzi A, Ahmadi Badi S, Moshiri A, Hasani-Ranjbar S, Soroush AR, Vaziri F, Fateh A, Ghanei M, Bouzari S, Najar-Peerayeh S, Siadat SD, Larijani B. Main gut bacterial composition differs between patients with type 1 and type 2 diabetes and non-diabetic adults.

23. Wei YS, Hsiao YC, Su GW, Chang YR, Lin HP, Wang YS, Tsai YT, Liao EC, Chen HY, Chou HC, Ko ML, Kuo WH, Chang SJ, Cheng WC, Chan HL. Identification of hyperglycemia-associated microbiota alterations in saliva and gingival sulcus. Arch Biochem Biophys. 2020 Mar 30;682:108278. J Diabetes Metab Disord. 2020 Feb 8;19(1):265-271. 
24. Wu M, Yang S, Wang S, Cao Y, Zhao R, Li X, Xing Y, Liu L. Effect of Berberine on Atherosclerosis and Gut Microbiota Modulation and Their Correlation in High-Fat Diet-Fed ApoE-/- Mice. Front Pharmacol. 2020 Mar 13;11:223.

25. Haghikia A, Li XS, Liman TG, Bledau N, Schmidt D, Zimmermann F, Kränkel N, Widera C, Sonnenschein K, Haghikia A, Weissenborn K, Fraccarollo D, Heimesaat MM, Bauersachs J, Wang Z, Zhu W, Bavendiek U, Hazen SL, Endres M, Landmesser U. Gut Microbiota-Dependent Trimethylamine $\mathrm{N}$-Oxide Predicts Risk of Cardiovascular Events in Patients With Stroke and Is Related to Proinflammatory Monocytes. Arterioscler Thromb Vasc Biol. 2018 Sep;38(9):2225-2235.

26. Pastori D, Carnevale R, Nocella C, Novo M, Santulli M, Cammisotto V, Menichelli D, Pignatelli P, Violi F. Gut-Derived Serum Lipopolysaccharide is Associated With Enhanced Risk of Major Adverse Cardiovascular Events in Atrial Fibrillation: Effect of Adherence to Mediterranean Diet. J Am Heart Assoc. 2017 Jun 5;6(6):e005784.

27. Yu L, Meng G, Huang B, Zhou X, Stavrakis S, Wang M, Li X, Zhou L, Wang Y, Wang M, Wang Z, Deng J, Po SS, Jiang H. A potential relationship between gut microbes and atrial fibrillation: Trimethylamine $\mathrm{N}$-oxide, a gut microbe-derived metabolite, facilitates the progression of atrial fibrillation. Int J Cardiol. 2018 Mar 15;255:92-98.

28. F, Prifti E, Falony G, Le Chatelier E, Levenez F, Doré J, Mattila I, Plichta DR, Pöhö P, Hellgren LI, Arumugam M, Sunagawa S, Vieira-Silva S, Jørgensen T, Holm JB, Trošt K; MetaHIT Consortium, Kristiansen K, Brix S, Raes J, Wang J, Hansen T, Bork P, Brunak S, Oresic M, Ehrlich SD, Pedersen 0. Human gut microbes impact host serum metabolome and insulin sensitivity. Nature. 2016 Jul 21;535(7612):376-81.

29. Tett A, Huang KD, Asnicar F, Fehlner-Peach H, Pasolli E, Karcher N, Armanini F, Manghi P, Bonham K, Zolfo M, De Filippis F, Magnabosco C, Bonneau R, Lusingu J, Amuasi J, Reinhard K, Rattei T, Boulund F, Engstrand L, Zink A, Collado MC, Littman DR, Eibach D, Ercolini D, Rota-Stabelli O, Huttenhower C, Maixner F, Segata N.The Prevotella copri Complex Comprises Four Distinct Clades Underrepresented in Westernized Populations.Cell Host Microbe. 2019 Nov 13;26(5):666-679.e7.

30. De Filippis F, Pellegrini N, Laghi L, Gobbetti M, Ercolini D.Unusual sub-genus associations of faecal Prevotella and Bacteroides with specific dietary patterns.Microbiome. 2016 Oct 21;4(1):57.

31. De Filippis F, Pasolli E, Tett A, Tarallo S, Naccarati A, De Angelis M, Neviani E, Cocolin L, Gobbetti M, Segata N, Ercolini D. Distinct Genetic and Functional Traits of Human Intestinal Prevotella copri Strains Are Associated with Different Habitual Diets.Cell Host Microbe. 2019 Mar 13;25(3):444453.e3.

32. De Filippis F, Pellegrini N, Vannini L, Jeffery IB, La Storia A, Laghi L, Serrazanetti DI, Di Cagno R, Ferrocino I, Lazzi C, Turroni S, Cocolin L, Brigidi P, Neviani E, Gobbetti M, O'Toole PW, Ercolini D. Highlevel adherence to a Mediterranean diet beneficially impacts the gut microbiota and associated metabolome.Gut. 2016 Nov;65(11):1812-1821.

33. Meslier V, Laiola M, Roager HM, De Filippis F, Roume H, Quinquis B, Giacco R, Mennella I, Ferracane R, Pons N, Pasolli E, Rivellese A, Dragsted LO, Vitaglione P, Ehrlich SD, Ercolini D. Mediterranean diet 
intervention in overweight and obese subjects lowers plasma cholesterol and causes changes in the gut microbiome and metabolome independently of energy intake. Gut. 2020 Jul;69(7):1258-1268.

\section{Tables}

Table 1. Baseline clinical characteristics, angiographic and procedural data in overall study-population. 


\begin{tabular}{|c|c|c|c|}
\hline & Hyperglycemic patients & Normoglycemic patients & $\mathbf{P}$ \\
\hline $\mathbf{N}$ & 56 & 90 & \\
\hline Mean age (years) & $64.1 \pm 6.8$ & $65.5 \pm 6.5$ & 0.204 \\
\hline $\mathrm{BMI}\left(\mathrm{kg} / \mathrm{m}^{2}\right)$ & $29.2 \pm 1.9$ & $29.7 \pm 1.9$ & 0.183 \\
\hline $\mathrm{SBP}(\mathrm{mmHg})$ & $119.9 \pm 8.1$ & $120.2 \pm 7.1$ & 0.809 \\
\hline $\mathrm{DBP}(\mathrm{mmHg})$ & $79.2 \pm 6.9$ & $79.3 \pm 6.4$ & 0.927 \\
\hline Heart rate $(\mathrm{bpm})$ & $87.6 \pm 9.1$ & $86.7 \pm 7.8$ & 0.515 \\
\hline \multicolumn{4}{|l|}{ LVEF } \\
\hline$>50 \%, \mathrm{n}(\%)$ & $46(82.1)$ & $69(76.7)$ & 0.284 \\
\hline $41 \%$ to $50 \%, n(\%)$ & 8 (14.3) & $17(18.9)$ & 0.315 \\
\hline $25 \%$ to $40 \%, n(\%)$ & $2(3.6)$ & $4(4.4)$ & 0.579 \\
\hline \multicolumn{4}{|l|}{ Laboratory analyses } \\
\hline Plasma glucose (mg/dl) & $202.4 \pm 27.3$ & $102.1 \pm 22.1$ & 0.006 \\
\hline Cholesterol (mg/dl) & $217.2 \pm 19.7$ & $205.5 \pm 23.1$ & 0.003 \\
\hline LDL-cholesterol (mg/dl) & $143.3 \pm 19.0$ & $132.2 \pm 22.8$ & 0.004 \\
\hline HDL-cholesterol (mg/dl) & $36.6 \pm 3.6$ & $47.2 \pm 3.4$ & 0.001 \\
\hline Triglycerides (mg/dl) & $196.8 \pm 23.1$ & $180.6 \pm 20.1$ & 0.0 .01 \\
\hline Creatinine $(\mathrm{mg} / \mathrm{dl})$ & $1.0 \pm .2$ & $1.0 \pm 0.2$ & 0.510 \\
\hline cTnT (ng/l) & $7.9 \pm 3.0$ & $7.6 \pm 1.2$ & 0.353 \\
\hline \multicolumn{4}{|l|}{ Risk Factors } \\
\hline Diabetes, n (\%) & $17(30.4)$ & $18(20)$ & 0.111 \\
\hline Hypertension, n (\%) & $35(62.5)$ & $56(62.2)$ & 0.558 \\
\hline Dyslipidemia, n (\%) & $16(28.6)$ & $29(32.2)$ & 0.392 \\
\hline Cigarette smoking, n (\%) & $3(5.4)$ & $8(8.9)$ & 0.366 \\
\hline New diabetes, n (\%) & $15(26.8 \%)$ & $16(10.9 \%)$ & 0.036 \\
\hline \multicolumn{4}{|l|}{ Active treatments } \\
\hline$\beta$-blockers, n (\%) & $28(50.0)$ & $32(35.6)$ & 0.061 \\
\hline ACE inhibitors, n (\%) & $25(44.6)$ & $31(34.4)$ & 0.156 \\
\hline Angiotensin receptor blockers, n (\%) & $165(16.5)$ & $372(37.2)$ & 0.145 \\
\hline
\end{tabular}




\begin{tabular}{|c|c|c|c|}
\hline Calcium inhibitor, n (\%) & $6(10.7)$ & $11(12.2)$ & 0.502 \\
\hline Statins, n (\%) & $15(26.8)$ & $25(27.8)$ & 0.527 \\
\hline Diuretic, n (\%) & $7(12.5)$ & $11(12.2)$ & 0.577 \\
\hline Insulin, n (\%) & $15(7.8)$ & $174(17.4)$ & 0.449 \\
\hline Oral anti-diabetic drugs, n (\%) & $16(28.6)$ & $25(27.8)$ & 0.667 \\
\hline Aspirin, n (\%) & $50(89.3)$ & $84(93.3)$ & 0.285 \\
\hline Thienopyridine, $\mathrm{n}(\%)$ & $5(8.9)$ & $5(5.6)$ & 0.321 \\
\hline \multicolumn{4}{|l|}{ Procedural data } \\
\hline Symptom to angiography, hours & $6.1 \pm 1.4$ & $6.0 \pm 1.1$ & 0.899 \\
\hline \multicolumn{4}{|l|}{ Angiographic data } \\
\hline \multicolumn{4}{|l|}{ Number of diseased vessels } \\
\hline 1-Vessel disease, n (\%) & $35(62.5)$ & $55(61.1)$ & 0.504 \\
\hline 2-Vessel disease, n (\%) & $15(26.8)$ & 30 (33.3) & 0.259 \\
\hline 3-Vessel disease, n (\%) & $5(8.9)$ & $5(5.6)$ & 0.321 \\
\hline \multicolumn{4}{|l|}{ TIMI Flow Grade } \\
\hline Grade 0, n (\%) & $13(23.2)$ & $25(27.8)$ & 0.341 \\
\hline Grade 1, n (\%) & $38(67.9)$ & $59(65.6)$ & 0.460 \\
\hline Grade 2/3, n (\%) & $5(8.9)$ & $7(7.8)$ & 0.517 \\
\hline Grade 3 post intervention, $\mathrm{n}(\%)$ & $53(94.6)$ & $86(95.6)$ & 0.546 \\
\hline \multicolumn{4}{|l|}{ Trombus Grade-Sianos et all. } \\
\hline G2 small, $n(\%)$ & $5(8.9)$ & $25(27.8)$ & 0.008 \\
\hline G3 medium, $n(\%)$ & $11(19.6)$ & $56(62.2)$ & 0.001 \\
\hline G4 large, $n(\%)$ & $30(53.6)$ & $8(8.9)$ & 0.001 \\
\hline G5 v. occlusion, $n(\%)$ & $10(17.9)$ & $1(1.1)$ & 0.001 \\
\hline \multicolumn{4}{|l|}{ Quantitative angiographic data } \\
\hline Lesion length, (mm) & $20.7 \pm 2.1$ & $20.1 \pm 3.2$ & / \\
\hline Reference diameter, (mm) & $2.7 \pm 0.5$ & $2.7 \pm 0.2$ & 0.579 \\
\hline MLD, (mm) & $1.0 \pm 0.1$ & $1.0 \pm 0.2$ & 0.755 \\
\hline MLD post (in-stent), (mm) & $2.6 \pm 0.3$ & $2.7 \pm 0.2$ & 0.290 \\
\hline
\end{tabular}




\begin{tabular}{|llll|}
\hline BMS, $\mathrm{n}(\%)$ & $10(17.9)$ & $15(16.7)$ & 0.511 \\
\hline DES, $\mathrm{n}(\%)$ & $46(82.1)$ & $75(83.3)$ & 0.499 \\
\hline Multivessel intervention, $\mathrm{n}(\%)$ & $18(32.1)$ & $28(31.1)$ & 0.578 \\
\hline
\end{tabular}

Data are means \pm SD or $\mathrm{n}(\%)$. BMI, body mass index, SBP, systolic blood pressure; DBP, diastolic blood pressure; LVEF, left ventricular ejection fraction; MLD, minimum luminal diameter; BMS, bare metal stent; DES, drug-eluting stent.

\section{Figures}

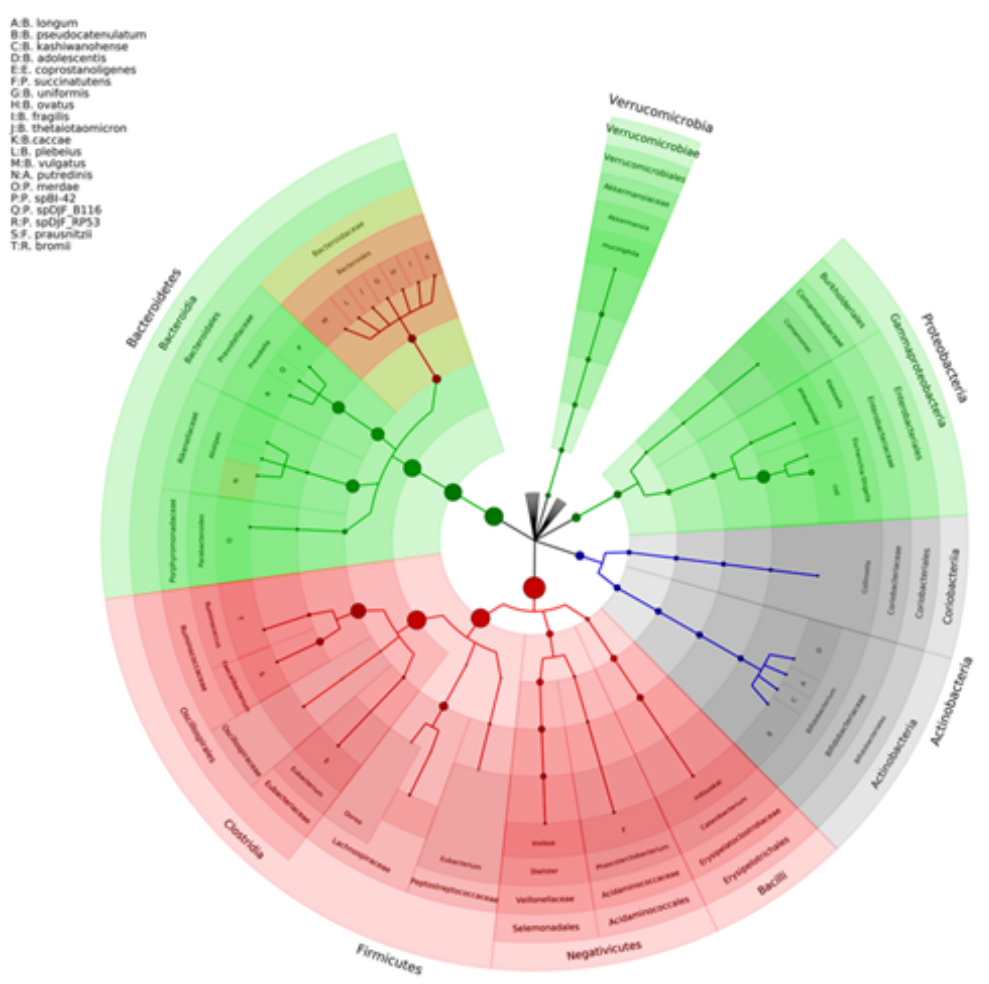

\begin{tabular}{|c|c|c|c|c|}
\hline Phylum & Class & Family & Species & ID \\
\hline Bacteroidetes & Bacteroidia & Rikenellaceae & Alistipes putredinis & $\mathbf{N}$ \\
\hline Bacteroidetes & Bacteroidia & Bacteroidaceae & Bacteroides caccae & $\mathbf{K}$ \\
\hline Bacteroidetes & Bacteroidia & Bacteroidaceae & Bacteroides fragilis & 1 \\
\hline Bacteroidetes & Bacteroidia & Bacteroidaceae & Bacteroides ovatus & $\mathbf{H}$ \\
\hline Bacteroidetes & Bacteroidia & Bacteroidaceae & Bacteroides plebeius & $\mathbf{L}$ \\
\hline Bacteroidetes & Bacteroidia & Bacteroidaceae & Bacteroides thetaiotaomicron & J \\
\hline Bacteroidetes & Bacteroidia & Bacteroidaceae & Bacteroides uniformis & G \\
\hline Bacteroidetes & Bacteroidia & Bacteroidaceae & Bacteroides vulgatus & M \\
\hline Bacteroidetes & Bacteroidia & Porphyromonadaceae & Parabacteroides merdae & $\mathbf{0}$ \\
\hline Bacteroidetes & Bacteroidia & Prevotellaceae & Prevotella sp. Bl-42 & $\mathbf{P}$ \\
\hline Bacteroidetes & Bacteroidia & Prevotellaceae & Prevotella sp. DJF B116 & $\mathbf{Q}$ \\
\hline Bacteroidetes & Bacteroidia & Prevotellaceae & Prevotella sp. DJF RPS3 & $\mathbf{R}$ \\
\hline Firmicutes & Clostridia & Ruminococcaceae & Faecalibacterium prausnitzii & $\mathbf{S}$ \\
\hline Firmicutes & Clostridia & Ruminococcaceae & Ruminococcus bromii & $\begin{array}{llllll}\mathbf{T} & \end{array}$ \\
\hline
\end{tabular}

\section{Figure 1}

Taxonomic Changes in Hyperglycemic Patients with STEMI. Phylogenetic tree showing differentiating taxa at the genus level between hyperglycemic and normoglycemic STEMI patients. The majority of depleted taxa in hyperglycemic microbiomes (red). Green indicates taxa enriched in hyperglycemic microbiomes. 


\section{THROMBUS MICROBIOTA}

A

PHYLUM

\#yperglycemic patients $\quad$ Normoglycemic patients

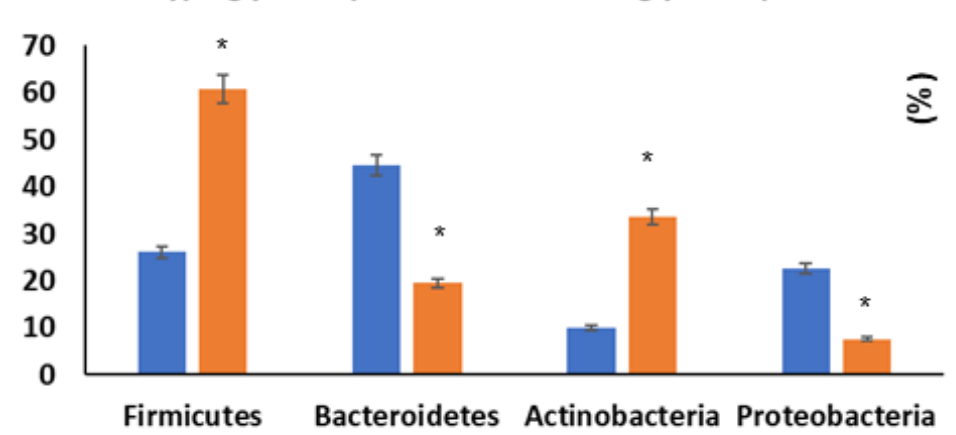

C

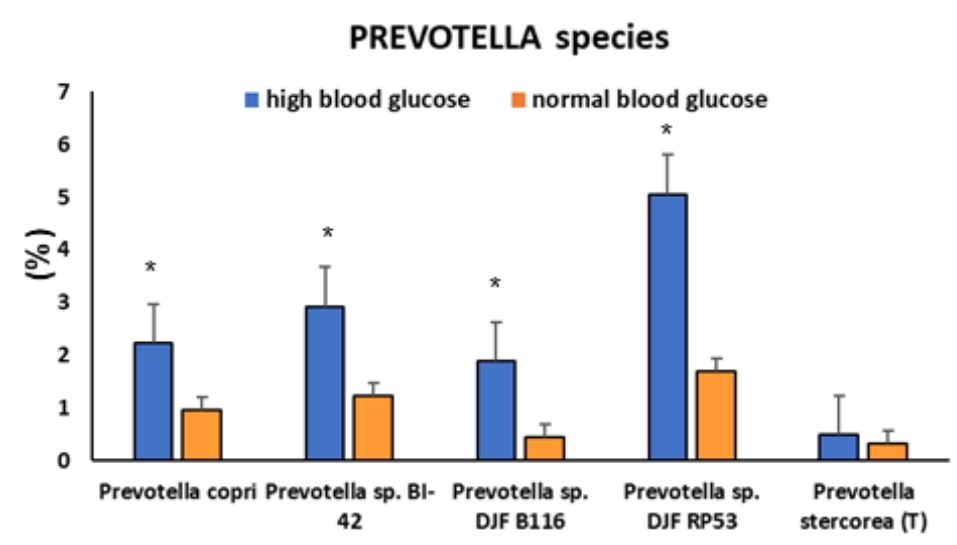

B

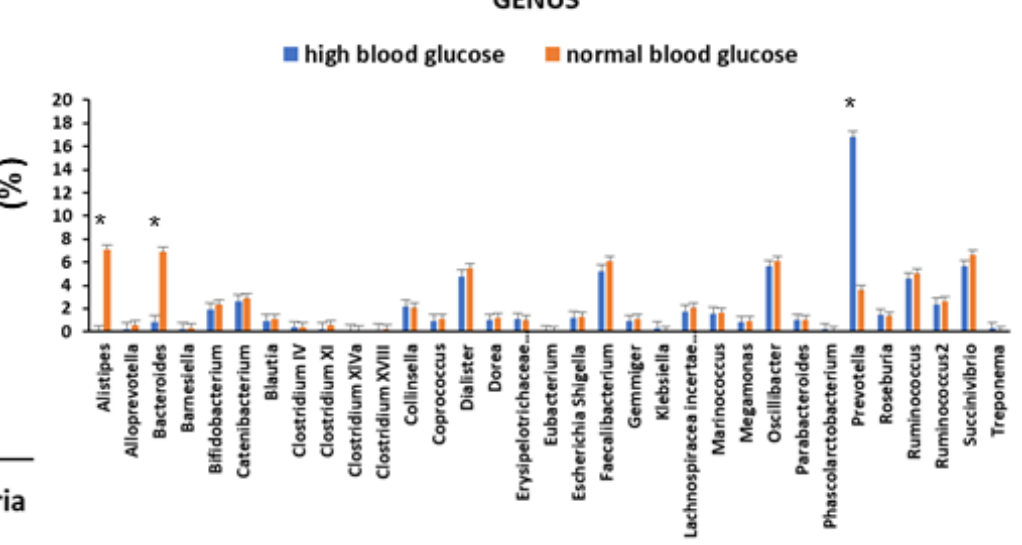

D

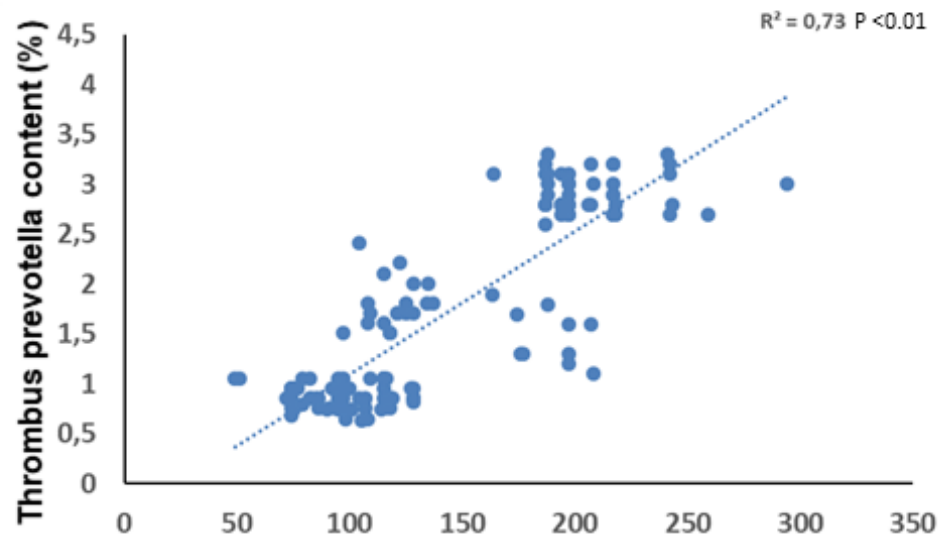

Admission blood glucose levels (mg/dl)

Figure 2

Thrombus Microbiota analysis. Panel A) filum, Panel B) genus, C) species in stool samples from hyperglycemic and normoglycemic STEMI patients. Panel D) regression analysis between admission blood glucose levels and thrombus Prevotella contents. 


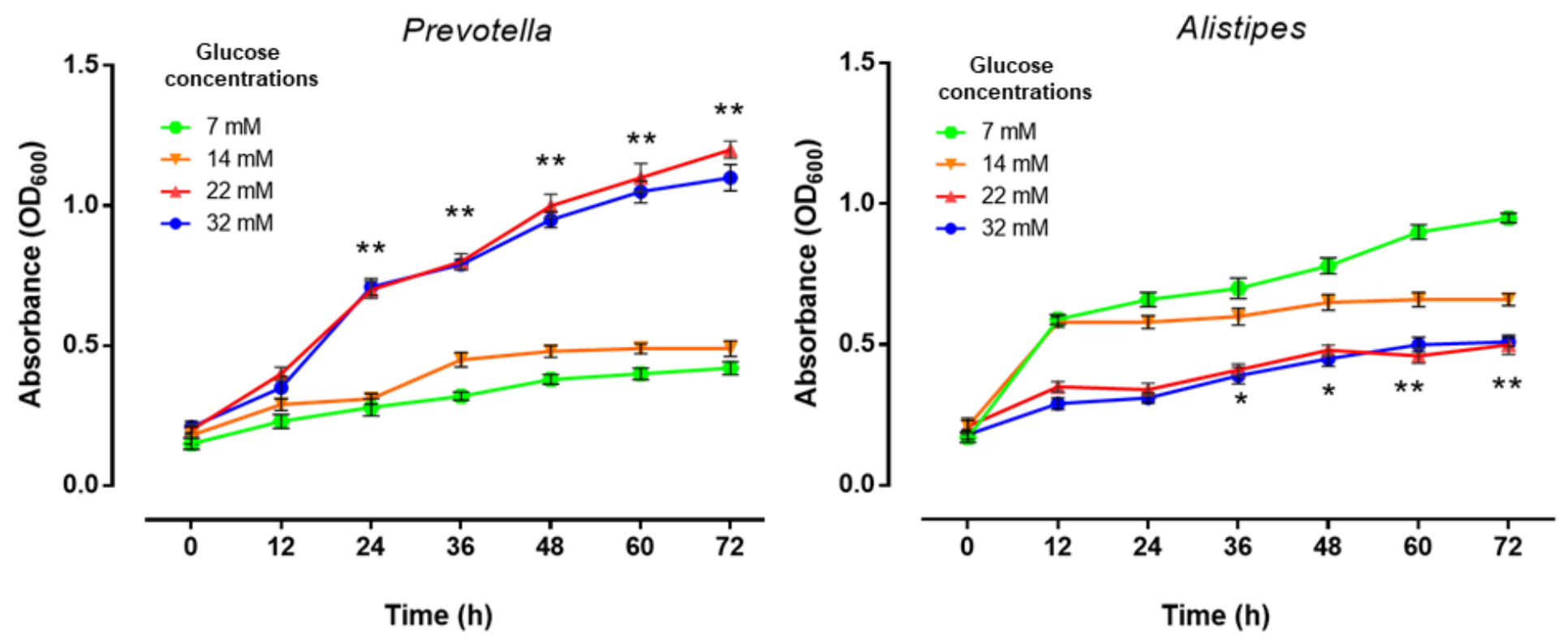

\section{Figure 3}

In vitro study. Panel A), Prevotella growth curve in normal and high glucose concentration culture. Panel $B)$, Alistipes growth curve in normal and high glucose concentration culture. ${ }^{*} P<0.05$ and ${ }^{* *} P<0.01$ vs $7 \mathrm{mM}$ glucose concentration. 


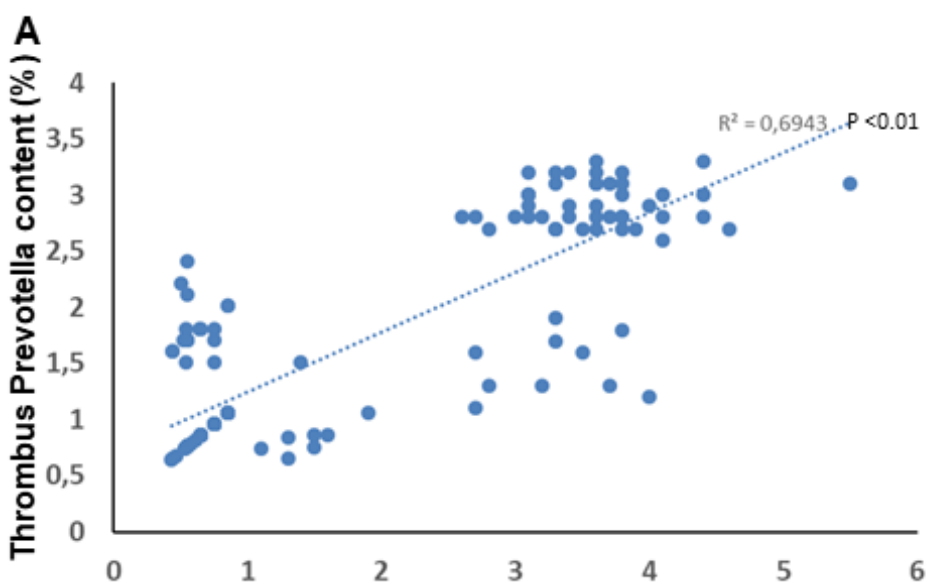

Thrombus dimension $\left(\mathrm{mm}^{2}\right)$

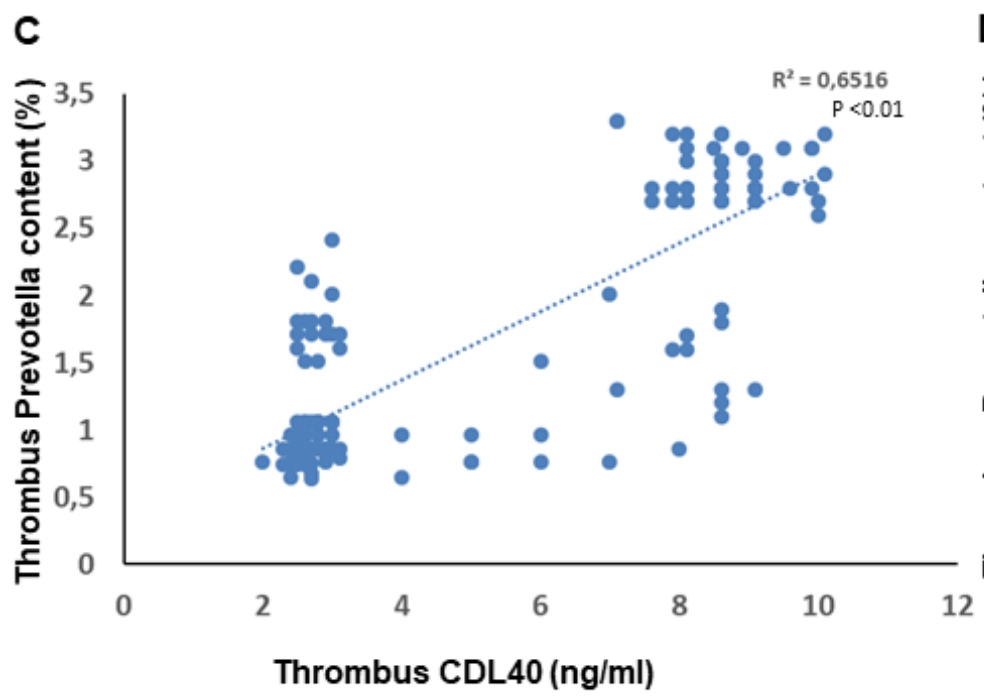

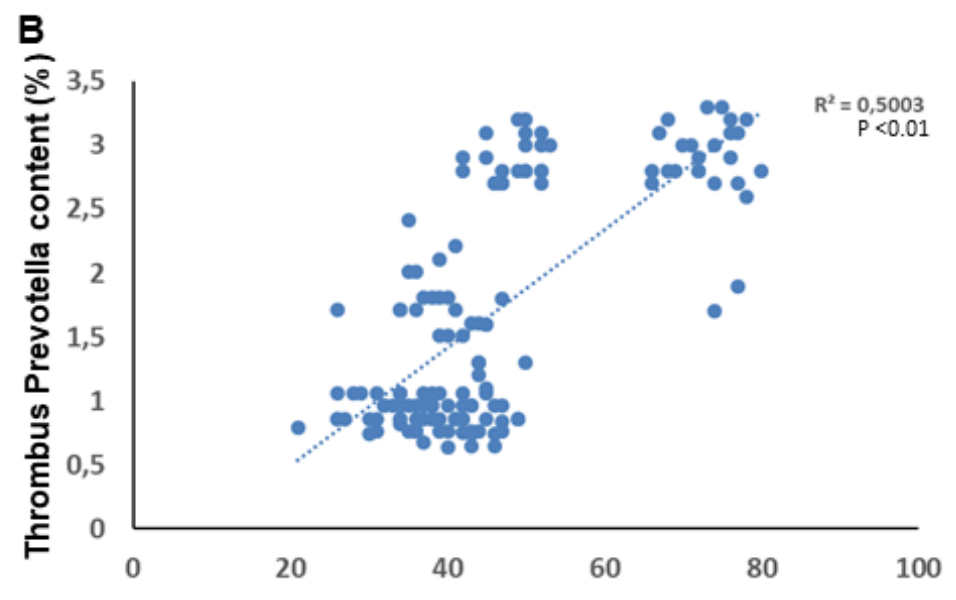

Thrombus TMAO content (ng/ml)

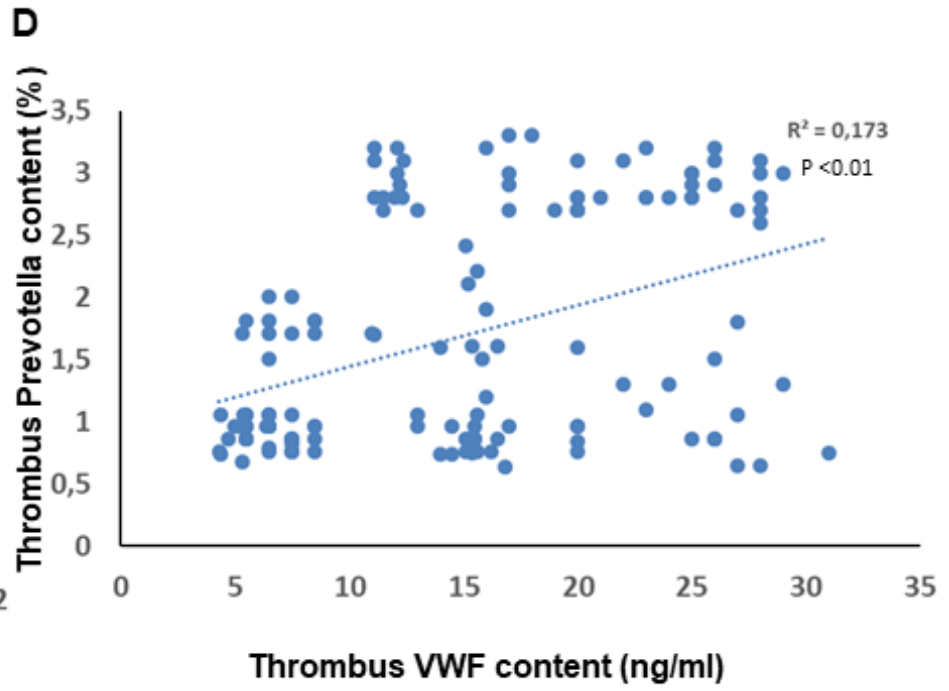

Figure 4

Regression analysis between thrombus Prevotella contents andthrombus dimensions (Panel A), TMAO thrombus levels (Panel B), CD40L thrombus levels (Panel C) and VWF thrombus levels (Panel D). 
A

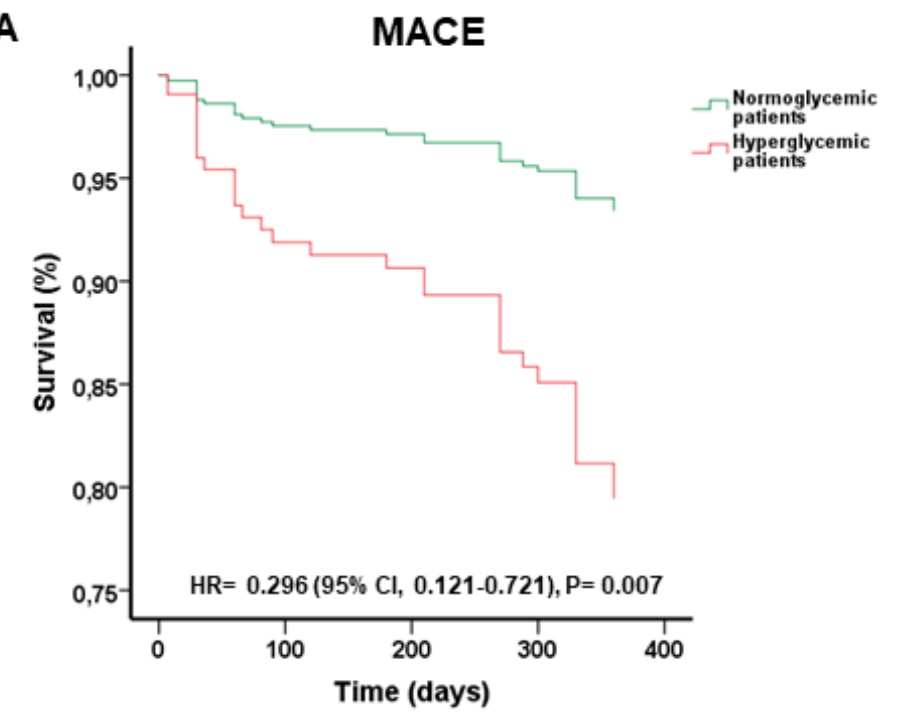

C

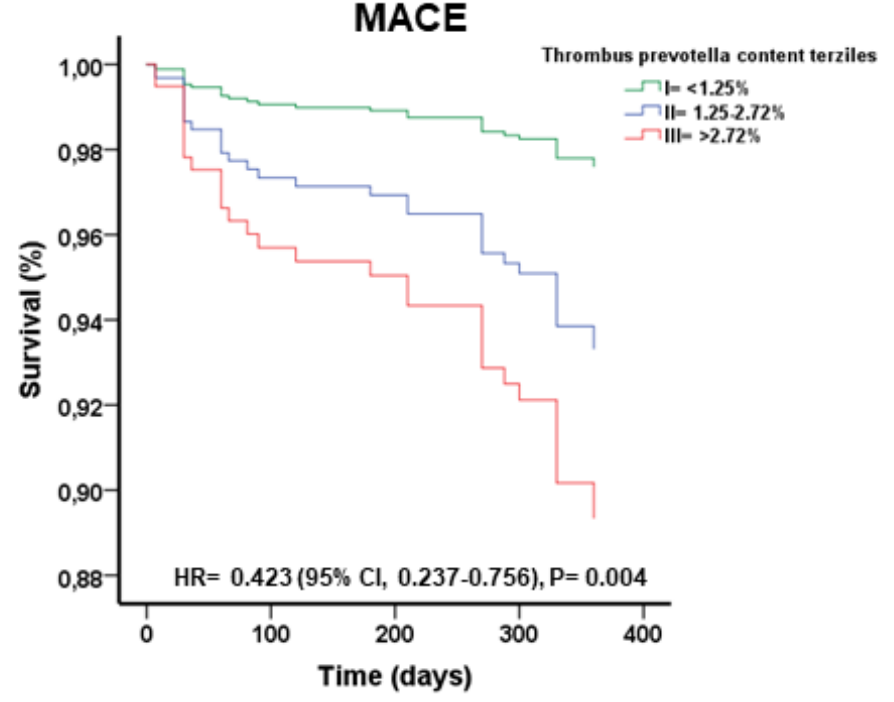

B

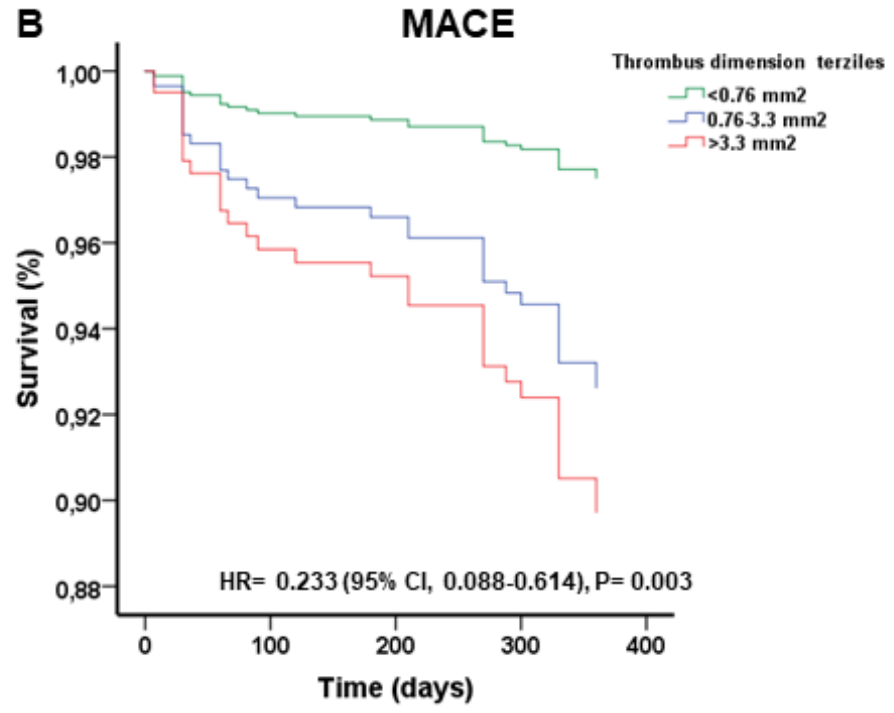

D

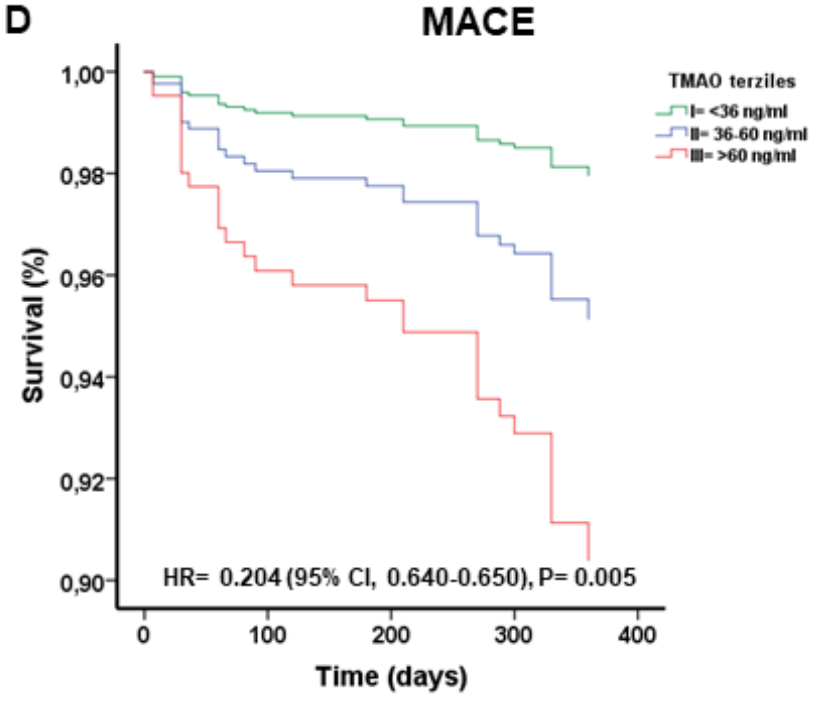

Figure 5

Risk adjusted Cox-regression analysis curves showing survival from MACE through 360 days in STEMI patients stratified by hyperglycemic vs normoglycemic patients (Panel A).Risk adjusted Cox-regression analysis curves showing survival from MACE through 360 days in STEMI patients stratified by thrombus dimension terziles (Panel B).Risk adjusted Cox-regression analysis curves showing survival from MACE through 360 days in STEMI patients stratified by thrombus Prevotella content terziles (Panel C). Risk adjusted Cox-regression analysis curves showing survival from MACE through 360 days in STEMI patients stratified by thrombus TMAO content terziles (Panel D). 


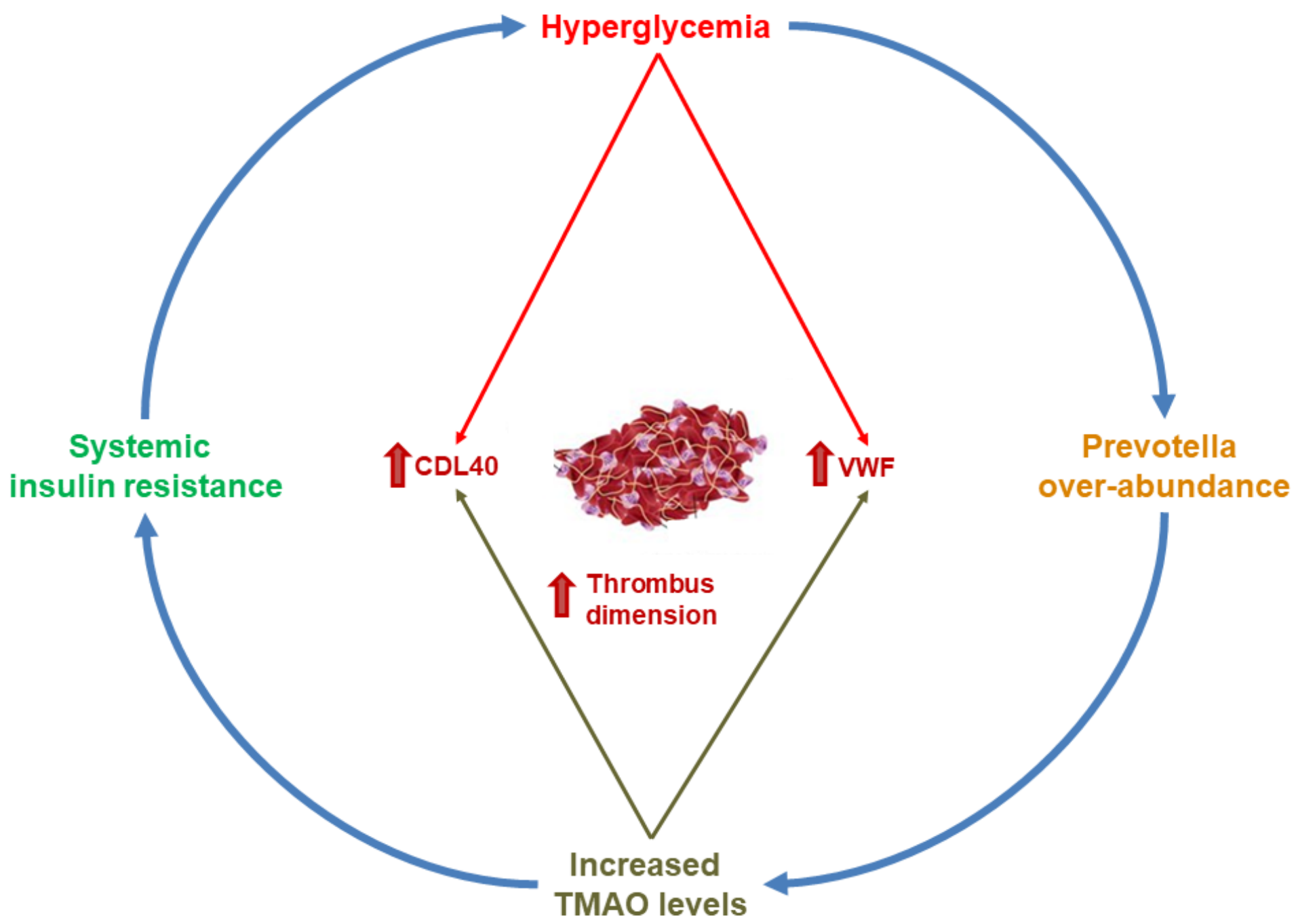

Figure 6

Hypothesis. 1) hyperglycemia promotes the growth and systemic colonization of Prevotella; 2) Prevotella overabundance increases TMAO levels; 3) high TMAO levels increase systemic insulin resistance; 4) increased insulin resistance increases hyperglycemia. Both hyperglycemia and high TMAO levels may increase CDL40 and VWF thrombus levels

\section{Supplementary Files}

This is a list of supplementary files associated with this preprint. Click to download.

- CVDSupplementaryFigures.docx

- SupplementaryFigure1.tif

- SupplementaryFigure2.tif

- SupplementaryFigure3.tif 